\title{
CRISPRa screening with real world evidence identifies potassium channels as neuronal entry factors and druggable targets for SARS-CoV-2
}

Authors: Chengkun Wang ${ }^{1, \dagger}$, Ravi K. Dinesh ${ }^{1, \dagger^{*},}$, Yuanhao Qu ${ }^{1,2,3, \dagger}$, Arjun Rustagi4, ${ }^{4,}$, Henry Cousins ${ }^{1,5, \neq}$, James Zengel ${ }^{6, \ddagger}$, Yinglong Guo ${ }^{7, \neq}$, Taryn Hall ${ }^{7, \ddagger}$, Aimee Beck ${ }^{4}$, Luke Tso $^{7}$, Elif Tokar Erdemic ${ }^{7}$, Kae Tanudtanud ${ }^{7}$, Sheng Ren ${ }^{7}$, Kathy Tzy-Hwa Tzeng ${ }^{7}$,

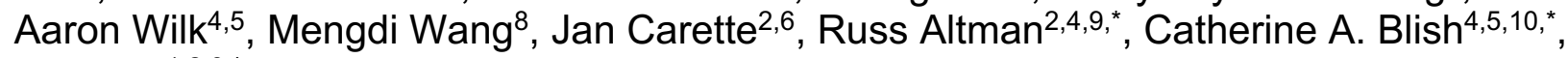
Le Cong ${ }^{1,2,3,{ }^{*}}$

\section{Affiliations:}

${ }^{1}$ Department of Pathology, Stanford University School of Medicine, Stanford, CA, USA

${ }^{2}$ Department of Genetics, Stanford University School of Medicine, Stanford, CA, USA ${ }^{3}$ Cancer Biology Program, Stanford University School of Medicine, Stanford, CA, USA ${ }^{4}$ Department of Medicine, Stanford University School of Medicine, Stanford, CA, USA ${ }^{5}$ Medical Scientist Training Program, Stanford University School of Medicine, Stanford, CA, USA

${ }^{6}$ Department of Microbiology and Immunology, Stanford University School of Medicine, Stanford, CA, USA

${ }^{7}$ Research and Development at UnitedHealth Group, Minneapolis, MN, USA

${ }^{8}$ Center for Statistics and Machine Learning, Department of Electrical and Computer Engineering, Princeton University, Princeton, NJ, USA

${ }^{9}$ Department of Bioengineering, Stanford University, Stanford, CA, USA

${ }^{10}$ Chan Zuckerberg Biohub, San Francisco, CA, USA

${ }^{*}$ Correspondence to: rdinesh@stanford.edu (R.K.D.); Russ.Altman@stanford.edu (R.A.); cblish@stanford.edu (C.A.B.); congle@stanford.edu (L.C.)

† These authors contributed equally to this work.

$\ddagger$ These authors contributed equally to this work. 


\begin{abstract}
Although vaccines for severe acute respiratory syndrome coronavirus 2 (SARS-CoV-2) have been successful, there are no good treatments for those who are actively infected and potentially suffer from diverse neurological symptoms. While SARS-CoV-2 primarily infects the respiratory tract, clinical evidence indicates that cells from sensory organs, brain, and heart are also susceptible to infection. An understanding of factors critical for viral infection in these tissues may help identify novel therapeutics. To discover host factors involved in SARS-CoV-2 viral entry, we performed CRISPR activation (CRISPRa) screens targeting all 6000+ human membrane proteins in cells with and without overexpression of ACE2 using Spike-pseudotyped lentiviruses. We identified both novel as well as previously validated host factors. Notably, we used replicationcompetent SARS-CoV-2 to validate new viral-entry promoting genes, including potassium channel KCNA6, protease LGMN, and MHC-II component HLA-DPB1. We found that the overexpression of KCNA6 led to a marked increase in infection even in cells with undetectable levels of ACE2 expression. Our analysis of human olfactory neuroepithelium scRNA-seq data revealed that OLIG2+ cells--previously identified as sites of infection in COVID-19 autopsy studies--have high KCNA6 expression and minimal levels of ACE2, suggesting that the presence of KCNA6 may explain sensory/neuronal aspects of COVID-19 symptoms. Further, we demonstrate that FDAapproved compound dalfampridine, an inhibitor of KCNA-family potassium channels, suppresses viral entry in a dosage-dependent manner. Finally, we identified common prescription drugs likely to modulate the top screen hits. We then performed a retrospective analysis of insurance claims of $\sim 8$ million patients and found a clinical association between screen-identified drug classes, particularly those targeting potassium channels, and COVID-19 severity. We have thus identified the potassium channel KCNA6 as a SARS-CoV-2 host factor, expanded our understanding of potential viral tropism, and identified promising targets for drug repurposing and development.
\end{abstract}




\section{Introduction}

The emergence of SARS-CoV-2 has led to the COVID-19 pandemic with over 150 million reported cases and over three million reported deaths as of May 2021 (WHO). Coronaviruses are a family of enveloped positive-stranded RNA viruses that cause respiratory and intestinal infections in birds and mammals ${ }^{1}$. Among the known human coronaviruses, four (229E, HKU1, NL63, and OC43) are widely circulating and cause mild infections, while three (SARS-CoV-1, Middle Eastern Respiratory Syndrome CoV, MERS-CoV, and SARS-CoV-2) are highly pathogenic ${ }^{1}$.

SARS-CoV-2 enters cells in three major steps. The virus Spike protein first binds to its canonical receptor, angiotensin converting enzyme 2 (ACE2). This is followed by proteolytic processing of the Spike which can be carried out by several proteases, with TMPRSS2 and Furin being the most well-known. These steps lead to membrane fusion and consequent release of viral RNA into the host cell ${ }^{2}$. Recent studies have also implicated the binding of Spike protein to heparan sulfate ${ }^{3}$ and cholesterol ${ }^{4-6}$ as well as soluble-ACE2-mediated host cell attachment ${ }^{7}$ as factors in viral entry, suggesting additional mechanisms that might be responsible for SARS-CoV-2 tropism and COVID19 pathology.

Several research groups performed CRISPR loss-of-function (LOF) screens to find factors necessary for SARS-CoV-2 entry and replication ${ }^{4,5,8-11}$. A large number of hits were only found in one or a few screens but not in others ${ }^{12}$. Further, several experimentally confirmed entry factors, most notably neuropilins ${ }^{13,14}$, were not reported as hits in any of the screens ${ }^{12}$. These discrepancies are likely due to the nature of LOF screens, which can only detect effects of genes that are expressed in the cell lines used.

To date one of the most efficacious treatments of SARS-CoV-2 infection remains antibody-based therapy that targets the SARS-CoV-2 Spike protein to inhibit ACE2 binding and prevent viral entry ${ }^{15}$. Similarly, current COVID-19 vaccines are highly effective in preventing symptomatic illness and function by triggering an immune response against the Spike protein ${ }^{16}$. However, newer viral strains with mutated Spike proteins have rendered both antibody therapies and vaccination potentially less effective ${ }^{17-21}$.

Taken together, this suggests a critical need to gain further insight into SARSCoV-2 entry mechanisms and develop therapeutics targeting this step in the viral lifecycle. We turned to CRISPR activation (CRISPRa) gain-of-function (GOF) screening of membrane proteins in cells with or without ACE2 receptor using Spike-pseudotyped lentiviruses. Our CRISPRa screen identified hits expressed in a broad spectrum of tissues, revealing previously unknown host factors in neuronal/sensory, respiratory, cardiovascular, and immune systems. We validated some of the most interesting candidate genes-including KCNA6, LGMN, and HLA-DPB1—using CDNA overexpression studies with both pseudoviral and replication-competent SARS-CoV-2 infection assays. Strikingly, we found that exogenous expression of the potassium channel KCNA6 promoted SARS-CoV-2 infection up to 50 fold in an ACE2independent fashion. After fixing issues with overlaps in genome annotation, we found that KCNA6 is highly expressed in OLIG2+ neuronal cells (shown to be infected in COVID-19 autopsy studies despite minimal ACE2/Neuropilin expression) in 
nasal/olfactory tissues ${ }^{13,22}$. We then performed a drug-target network analysis and a large retrospective study analyzing insurance claims data, which provided evidence for a clinical association between drug classes targeting our CRISPRa screen hits, especially potassium channels, and COVID-19 severity.

\section{Results}

\section{Membrane-focused CRISPRa screens identify putative host factors determining susceptibility to SARS-CoV-2.}

Our CRISPRa screening integrated a dCas9 synergistic activation mediator (SAM) system, a SARS-CoV-2 Spike-D614G pseudoviral entry assay, and a single guide (sgRNA) library targeting all known human membrane proteins (Fig. 1A, fig. S1A). Specifically, to discover host factors that promote ACE2-dependent and independent entry of SARS-CoV-2, we engineered two HEK293FT cell lines with or without ACE2 expression (ACE2-null and ACE2-positive) (fig. S1B). We then engineered them to express the SAM system (Fig. 1A, fig. S1A) and confirmed the gene activation efficiencies of the cell lines (fig. S1C). We next generated a pseudovirus-based SARS-CoV-2 entry assay as our functional read-out using a Spikepseudotyped lentivirus carrying a Zeocin resistance marker fused to a GFP reporter, and confirmed ACE2-dependent pseudoviral infection (fig. S1D). Of note, we chose to use SARS-CoV-2 Spike protein with the D614G mutation to address the dominance of D614G in global circulating viral sequences. We generated a customized CRISPRa sgRNA library targeting all known human membrane proteins ( 6,213 genes totaling $\sim 24,000$ sgRNAs with non-targeting controls), and made sure to include predicted membrane proteins that are sometimes absent in genome-wide libraries but could be hidden host factors. We then performed the screening workflow using pseudovirus carrying either SARS-CoV-2 Spike protein (SARS-CoV-2 group) or vesicular stomatitis virus envelope $G$ protein (VSVG group, as a reference) (Fig. 1A).

We performed the full CRISPRa screen across four conditions: ACE2-null or ACE2-positive at either high or low multiplicity of infection (MOI). We generated at least two biological replicates for each condition and integrated replicates into gene enrichment summaries (Fig. 1B-E). The results demonstrated that our approach could identify factors known to promote Spike-dependent viral entry (e.g., ACE2, TMPRSS2, Neuropilin) as well as new genes and pathways (e.g., ion channels, immune and neuronal receptors). First, several genes in the ACE2-null group had significant enrichment that implicated ACE2-independent mechanisms of viral entry (Fig. 1B-C, fig. S2). Among these genes, some were within known pathways, such as heparan sulfate synthesis enzymes EXT1 and EXTL2 ${ }^{3}$. Besides known host factors, several ion channel, protease, and immune genes were consistently ranked as top hits, e.g. KCNA6 (a potassium channel), LGMN (a lysosomal/endosomal protease), and HLA-DPB1 (MHC class II beta chain) (Figure 1F-G). Second, for the ACE2-positive groups, our screens identified known host factors, including TMPRSS family and NDST enzymes, as well as the recently discovered ACE2-potentiating factor Neuropilin (Fig. 1D-E, fig. S2). NRP2, in particular, was strongly enriched in the ACE2-positive group. Additionally, several ion channels and neuronal receptors (sodium and potassium channels, GABA 
receptors) were enriched in this group. A few immune-related genes, such as CD7 and HLA-DPB1, were also among top hits in the presence of ACE2.

\section{Analyses of top-ranked screen hits identify host pathways involved in SARS-CoV- 2 infection and reveal a prominent role for potassium channel genes in viral entry.}

To examine the top-ranked screen hits, we conducted three analyses: (1) differential analysis compared with VSVG reference screens and previous CRISPR loss-of-function screening studies; (2) tissue expression analysis; and (3) functional pathway enrichment analysis.

First, we sought to identify host factors that specifically promoted SARS-CoV-2 Spike-dependent entry. We ran parallel reference screens using VSVG-pseudotyped lentivirus. As expected, we identified robust enrichment of low-density lipoprotein receptor (LDLR) family members--the canonical VSV host receptors ${ }^{23}$--among the top hits in the VSVG groups, including LDLR, LDLRAD3, and LRP4/8 (Fig. 1F-G). In contrast, known SARS-CoV-2-specific host factors, such as ACE2 and TMPRSS2, were unique to the SARS-CoV-2 screens (Fig. 1F-G). Critically, the VSVG reference allowed us to rule out highly enriched genes in SARS-CoV-2 Spike screens that were nonspecific. These genes included known non-specific factors involved in apoptosis and growth, such as BAX/PKN2, fatty acid biosynthesis genes PIGO/PIGP/FADS6, and endocytosis/exocytosis genes GULP1/SCARA5. Other potentially pan-lentiviral or panviral factors were the integrin ITGAX, and two genes of the P4-ATPase Flippase Complex (the alpha subunit ATP10D, and the beta subunit TMEM30A). In sum, the use of a VSVG reference allowed us to narrow down the range of candidate factors and determine top hits that are specific to SARS-CoV-2 Spike (Fig. 1F-G).

In addition, we compare our CRISPRa screens with six recently published CRISPR LOF screens. We extracted the relative rankings of 4923 shared membrane proteins, and generated a curated gene list of 18 validated virus entry factors reported by published studies. We examined the ability of each CRISPR screen to identify these virus entry factors (fig. S3A). We found that 12 out of the 18 reported entry factors were among the top $10 \%$ of the highest ranked genes from our screens (fig. S3).

Second, we examined the expression of top-ranked genes across 24 tissues using the Genotype-Tissue Expression (GTEx) dataset ${ }^{24}$ (Fig. 2A). Several hitsincluding STOM, LGMN, and TSPAN15-were broadly expressed across tissues while others showed tissue-specific expression. For example, TMPRSS family genes and SLC26A9 were enriched in the respiratory tract (esophagus and lung), the primary site of SARS-CoV-2 infection. We found that many hits showed high expression levels in neuronal, cardiovascular, liver, and gastrointestinal systems, four organs that have been shown to be involved in the pathophysiology of SARS-CoV-2 ${ }^{25-27}$ (Fig. 2B). Notably, a significant number of our highly-ranked genes are ion channels, transporters or receptors expressed in the brain and sensory systems, including ASIC1, EPHA4, GABRB2, KCNA6, LRRC8D, RDH10, and SCNN1D. Of note, KCNA6 is ranked consistently at top in our screens, but is absent in the latest GTEx database due to overlapping annotations, so we had to study its expression using an alternative source ${ }^{28}$. Further, we identified liver-enriched hits (e.g. CLEC4G, SIGMAR1) and cardiovascular-enriched hits (e.g. CYSLTR2 and ISLR). Intriguingly, our top hits 
included several genes highly expressed in immune cells, such as CD7 and MHC-II components. Although there is no direct evidence that immune cells are susceptible to SARS-CoV-2 infection, prior studies have detected SARS-CoV-2 RNA in immune cell types ${ }^{29}$. Our results potentially explained the presence of SARS-CoV-2 in diverse tissues reported to be susceptible to SARS-CoV-2 ${ }^{25-27 .}$

Third, we performed functional enrichment and network analyses using topranked screen hits. Gene ontology and pathway enrichment (Reactome) analyses identified several biological processes that might relate to SARS-CoV-2 infection (Fig. 2C, fig. S4A). Some were known processes involved in SARS-CoV-2 virus entry, including glycosylation, heparan sulfate synthesis, and peptidase/protease activities ${ }^{3,30}$. Moreover, some previously unappreciated biological processes ranked highly in the functional analysis, specifically ion channels (potassium, sodium) and immune receptor activities (Fig. 2C). To look more closely into these processes, we built detailed functional networks (Reactome FI) using hits within the top-ranked biological processes (Fig. 2D, fig. S4F) or top screen hits (fig. S4B-E). One prominent gene family present is the voltage-gated potassium channel (Kv channel) family (Fig. 2D). This enrichment of potassium channels and other genes of interest within a functional group pointed to specific host pathways that may mediate or facilitate SARS-CoV-2 entry.

\section{Pseudoviral and replication-competent SARS-CoV-2 infection validation directly links the expression of top screen hits, most prominently KCNA6, with enhanced viral entry.}

As part of the initial validation of our screens, we created a subpool CRISPRa library targeting top-ranked genes, transduced them into ACE2-null and ACE2-positive cells, and assayed them with both wild-type (WT) and D614G Spike-pseudotyped lentiviruses (fig. S5). These experiments confirmed consistent performance of topranked sgRNAs irrespective of the use of WT or D614G Spike-pseudotyped lentiviruses (fig. S5). To validate individual top-ranked genes, we generated focused CRISPRa cell lines in arrayed format and tested them with GFP reporter pseudoviral infection. Activation of high-ranking genes, such as KCNA6, SCNN1D, LGMN, CD7 and MHC-II genes, in ACE2-null cells strongly promoted pseudoviral infection (fig. S6A). The effects in ACE2-positive cells were less prominent due to ACE2 overexpression, but activation of either LGMN or NRP2 promoted pseudoviral entry at levels comparable to TMPRSS2 (fig. S6B).

Next, we generated cDNA overexpression cell lines for selected high-ranking genes in ACE2-null and ACE2-positive conditions to directly connect target protein expression with viral entry promotion (Fig. 3A-B, fig. S7,8). We performed arrayed validation on cDNA overexpressing lines using Spike-D614G and VSVG pseudotyped lentivirus. The results of these cDNA experiments differed from the CRISPRa validation, helping to identify bona fide entry-promoting effects as unequivocal consequences of exogenous gene expression (Fig. 3A-B). In the ACE2-null condition, overexpression of KCNA6 or CD7 specifically promoted Spike-mediated pseudoviral infection, with KCNA6 expression increasing viral entry $\sim 4$-fold above the control, compared with $\sim 15$-fold for ACE2 cDNA (Fig. 3A). In the ACE2-positive condition, overexpression of almost all CDNAs enhanced pseudovirus entry. CD7, EPHA4, LRCC8D, LGMN, and RDH10 
overexpressing ACE2-positive lines showed over 2-fold increases in Spike-mediated infection over the control, and these effects were not seen for VSVG pseudovirus (Fig. 3B). Additionally, we used quantitative PCR (qPCR) and Western Blotting to verify cDNA expression in cell lines that promoted Spike-dependent viral entry most robustly (fig. S9).

Using replicating Spike-pseudotyped VSV with time-lapse imaging of virus infection, we found that the strongest hits validated by the pseudotyped lentiviral experiments, such as CD7, KCNA6, and LGMN, were able to similarly promote infection (Fig. 3C-D). Parallel experiments with Rabies virus (RABV) G protein pseudotyped VSV in the same cell lines showed no cDNA-dependent effects (fig. S10). The consistent results from the two pseudoviral systems confirmed that effects from our genes of interest were specific to SARS-CoV-2 Spike, and not artifacts of the systems used.

Finally, we used a nanoluciferase-expressing SARS-CoV-2 reporter virus, icSARS-CoV-2nLuc ${ }^{31}$, to measure if our candidate overexpression lines could promote live virus infection. Consistent with our pseudovirus results, KCNA6 and LGMN significantly promoted SARS-CoV-2 infection compared with control groups in ACE2null cells (Fig. 3E). Notably, in the ACE2-null condition, KCNA6 overexpression led to a $\sim 50$-fold increase in SARS-CoV-2 virus entry, compared with a 150 -fold increase in the ACE2 positive control (Fig. 3E). To our knowledge, KCNA6 is the strongest ACE2independent host factor for SARS-CoV-2 that has been validated by replicationcompetent virus infection. In addition, KCNA6, HLA-DPB1, EPHA4, CD7, and LGMN also had the ability to promote viral infection in the ACE2-positive condition (Fig. 3F). These results demonstrated that our findings were translatable to replication-competent SARS-CoV-2 biology.

\section{KCNA6 is highly expressed in nasal/olfactory neurons located at sites of SARS- CoV-2 infection and pathology.}

KCNA6 was the strongest candidate host factor from our replication-competent SARS-CoV-2 validation, but it had not been identified in previous studies as a viral entry factor. We sought to understand why KCNA6 had been overlooked and investigated its expression in human tissues to gain insight into its role in viral susceptibility.

As noted earlier, KCNA6 is missing from the current GTEx database due to genome annotation overlaps in the latest GRCh38/hg38 genome reference, which may explain its absence in prior studies. So we turned to a bulk RNA-Seq dataset ${ }^{32}$ where KCNA6 expression was detected with the usage of an older genome reference, GRCh37/hg19. From this analysis, we found that KCNA6 has uniquely high expression in the olfactory neuroepithelium and brain, unlike the canonical SARS-CoV-2 receptor ACE2 (Fig. 4A). We then looked into KCNA6 expression in single-cell RNA-seq (scRNA-seq) analysis. Given almost all commonly used scRNA-seq pipelines-such as 10x CellRanger, STARsolo, and Kallisto-discard multiple-mapped reads, we found that KCNA6 expression was nearly undetectable in published single-cell studies when using the latest genome reference GRCh38/hg38 (Fig. 4B-C).

Specifically, the overlapping annotations in GRCh38 led to KCNA6 being either discarded from the annotation (the case for GTEx), or with no aligned reads (the case 
for scRNA-seq pipelines). All three overlapping features are non-coding: two long noncoding RNAs (RP11-234B24, RP3-377H17, non-conserved in mammals) and a processed non-coding transcript of nearby GALNT8. The length of KCNA6 annotation is also shorter in GRCh38 vs. GRCh37, as the KCNA6 untranslated region (UTR) in GRCh37 was re-assigned to non-coding features in GRCh38 (Fig. 4B). Thus, the GRCh38 annotation makes it difficult to measure KCNA6 expression even using pipelines that can handle multiple-mapped reads. We re-processed scRNA-seq data of olfactory neuroepithelium from two patients ${ }^{33}$. Indeed, due to annotation issues, CellRanger and the multimapping-aware Salmon-Alevin pipelines could not detect KCNA6 expression using the GRCh38 reference (Fig. 4C-D). After adjusting the genome reference to remove the overlapping transcripts, we detected KCNA6 expression in olfactory/nasal tissue samples from human patients using the CellRanger pipeline (Fig. 4C). The Salmon-Alevin pipeline can handle multiple-mapped reads, and allowed us to readily detect high levels of KCNA6 expression with the standard GRCh37 reference (Fig. 4D).

Using this updated genome reference, we analyzed single-cell expression of KCNA6 in the nasal/olfactory neuroepithelium. Neurons in this tissue were reported to be infected and involved in COVID-19 pathology, but have very low expression of ACE2, Neuropilins, or other known entry factors ${ }^{34-36}$. We found KCNA6 is robustly expressed in neuronal cells within human olfactory neuroepithelium (Fig. 4E-F). Further, we detected high expression of KCNA6 in OLIG2+ neurons (Fig. 4G).

Two recent studies using COVID-19 autopsy samples have demonstrated that SARS-CoV-2 is detected in OLIG2+ neurons within patient olfactory epithelium ${ }^{13,22}$. Our analysis showed KCNA6 is uniquely present in this virus-infected cell type (Fig. $\mathbf{4 H}$ ), which can only be seen using the updated genome reference. Our findings suggest the presence of KCNA6 could help explain sensory and neuronal aspects of COVID-19 symptoms and suggest the potential for uncovering new SARS-CoV-2 entry mechanisms ${ }^{37}$.

\section{Potassium channel inhibition using an FDA-approved compound suggests KCNA6 is a drug target for SARS-CoV-2.}

As potassium channels are druggable targets, we tested an FDA-approved potassium channel blocker, 4-Aminopyridine (4-AP, dalfampridine), for its ability to suppress viral entry (Fig. 4I). 4-AP has broad inhibitory activity against potassium channels, most prominently targeting the KCNA-family ${ }^{38}$. We observed that 4-AP inhibited SARS-CoV-2 pseudovirus entry in a dosage-dependent manner (Fig. 4J-K). In ACE2-null groups, 4-AP blocked Spike-mediated viral entry due to KCNA6 expression, with an $I_{50}$ of $\sim 490$ uM (Fig. 4J). This effect is specific to SARS-CoV-2 as 4-AP had no effect on VSVG pseudoviral infection (Fig. 4J). In ACE2-positive groups, 4-AP suppressed SARS-CoV-2-Spike pseudovirus entry in both the BFP and the KCNA6 lines, but again had no effect on VSVG pseudoviral infection (Fig. 4K). Here, we observed that KCNA6 overexpression mitigated the inhibitory effects of 4-AP (IC 50 of 906uM in BFP control vs. 1095uM in KCNA6 cDNA line) (Fig. 4K). The modest effect size here was likely due to the significantly lower levels of KCNA6 protein in ACE2positive vs. ACE2-null cells (fig. S9A). Overall, these results suggest targeting 
potassium channels, particularly KCNA6, as a promising avenue of COVID-19 treatment independent of ACE2-mediated pathways.

\section{The protease LGMN and MHC-II gene HLA-DPB1 are potential host factors for SARS-CoV-2 infection.}

From our pseudoviral and replication-competent SARS-CoV2 validation, overexpression of the protease LGMN and the major histocompatibility complex (MHC) Class II component HLA-DPB1, increased viral entry significantly (Fig. 3B,F). LGMN, which encodes human legumain/delta-secretase, is thought to be a lysosomal/endosomal asparaginyl endopeptidase (AEP) with broad tissue distribution, and may be activated in an age-dependent manner ${ }^{39}$. HLA-DPB1 is a human leukocyte antigen (HLA) class II beta chain protein expressed in many human tissues, including the lung. Thus, we examined their single-cell gene expression profiles from published lung bronchoalveolar lavage fluid (BALF) samples of COVID-19 patients ${ }^{29}$. Our metaanalysis indicated that both LGMN and HLA-DPB1 expression had a positive correlation with levels of SARS-CoV-2 viral RNA (fig. S11). Moreover, we observed that LGMN expression was positively correlated with COVID-19 disease severity (fig. S11C). Taken together, LGMN and HLA-DPB1 could be potentially involved in host cell susceptibility to SARS-CoV-2 and COVID-19 disease progression.

\section{Retrospective analysis of patient claims provides real-world evidence that common drugs targeting potassium channels may lower risks of COVID-19 hospitalization.}

As our screen targeted all human membrane proteins, the majority of our hits are within the druggable genome. We next asked whether existing drugs are likely to modulate host factors identified in our CRISPRa screens. We constructed a bipartite graph representing known interactions between 4,929 FDA-approved compounds and 2,325 protein-coding genes, 254 of which we identified as SARS-CoV-2-specific screen hits (Fig. 5A). To evaluate which drugs were likely to modulate viral entry, we ranked compounds by normalized degree centrality (NDC) with respect to screen hit genes, and identified enriched drug categories by median NDC. We observed marked enrichment of several drug classes with a propensity for potassium-channel targeting, including antidepressant, anticonvulsant, and antipsychotic agents (Fig. 5B, left). Many of these classes have previous literature support for a role in modulating SARS-CoV-2 viral entry 40,41 . To assess the specificity of the highest-ranking drug classes relative to their entire interaction profile, we further scored them by the proportion of drug interactors that were CRISPRa screen hits (degree ratio). This approach identified a similar set of ion-channel-targeting drug categories (Fig. 5B, right).

Next, to provide a clinical assessment of our findings, we performed a retrospective analysis of claims data from a large US health insurance provider, examining associations between common prescriptions and COVID-19 hospitalization rates. We reviewed claims from 7.8 million Medicare Advantage Part D (MAPD) members for compatibility with regional and temporal inclusion criteria (Fig. 5C). The final dataset comprised claims for 234,524 MAPD-insured individuals, with at least 11 
months of enrollment between January and December 2019 and at least one month of enrollment during 2020, with at least one pharmacy prescription claim from the UnitedHealth Group Clinical Discovery Portal. Among these individuals, 2,828 (1.21\%) had claims indicating COVID-19 hospitalization during the observation window.

We screened the claims database for commonly prescribed drugs associated with COVID-19 hospitalization in a 1:10 matched cohort of hospitalized/non-hospitalized patients. We identified 98 drugs whose odds ratio was significant (corrected $p<0.05$ ). This included drugs in several mechanistic classes: broad-spectrum anticonvulsants, angiotensin-converting enzyme (ACE) inhibitors, angiotensin II receptor antagonists, and thiazide diuretics. These drugs were highly ranked in the drug-target network derived from our screen hits ( $p<1 e^{-11}$, Mann-Whitney $U$ test; Fig. 5D), suggesting an association between a compound's ability to modulate viral entry genes and its associated risk of COVID-19 hospitalization.

We selected four drug classes for follow-up analysis using 1:1 propensity score matching (PSM). The classes, which included loop diuretics, opioid analgesics, selective serotonin reuptake inhibitor (SSRI) antidepressants, and broad-spectrum anticonvulsants, were ranked highly in the drug-target network, had sufficient prescription volume in the claims dataset, and included known potassium-channeltargeting drugs. The hazard ratios for opioid analgesics were not significant either before or after PSM matching. We observed a significant risk-associated effect for loop diuretics, SSRI antidepressants, and broad-spectrum anticonvulsants that persisted after PSM, with hazard ratios $>1.2$ consistently and significantly (Fig. 5E).

Further, we investigated angiotensin pathway genes, including KCNA channels, which were the most prominent and well-validated category of hits in our experiments. We evaluated whether common drugs targeting these ion channels were associated with decreased risk of hospitalization. Our clinical analysis showed that the diuretic hydrochlorothiazide (HCTZ) alone or in combination with ACE inhibitors (ACEi) were consistently associated with a protective effect, even when compared to controls on other first-line antihypertensive agents, with hazard ratios between 0.72 0.85 and statistical significance (Fig. 5F).

\section{Discussion}

In the work presented here we demonstrate the utility of CRISPRa screening to provide insight into the tropism of an emerging pathogen. CRISPR LOF approaches are powerful, but limit discovery of host factors to genes expressed within the context of the cell line used. A potential path to overcome these limitations is doing screens in multiple cell lines representing a variety of cell types, but this is laborious and necessitates prior knowledge of viral tropism. Our CRISPRa screening approach overcomes these limitations by selecting cell lines with limited or no susceptibility and allowing for the unbiased determination of factors that promote viral entry. We show here that previously unknown factors in a diverse set of tissues-such as neuronal (KCNA6), immune (HLADPB1, CD7), and cardiac (LGMN, EPHA4)—can stimulate SARS-CoV-2 viral entry.

Most strikingly, we show that KCNA6, a voltage-gated potassium channel, potentiates SARS-CoV-2 entry in a cellular context where ACE2 expression is 
undetectable (Fig. 3E, 4H). Further, our additional tests demonstrated that, under such ACE2-null conditions, KCNA6 overexpression promoted pseudovirus infection when using different variants of the SARS-CoV-2 Spike, including a variant of concern B.1.351 (Beta variant, first identified in South Africa) (Fig. S12A). With minimal ACE2 expression, the Spike variants D614G and B.1.351 supported comparable ability as wild-type Spike to infect KCNA6-overexpressing cells in pseudoviral assay (Fig. S12B). This could be significant in light of reports that emerging SARS-CoV-2 variants are often less dependent on ACE2 binding, and resistant to antibody therapeutics ${ }^{17,19,42}$.

KCNA6 is highly expressed in OLIG2+ neuronal cells (Fig. 4F-H), which have been shown to be a cell type susceptible to SARS-CoV-2 infection in the olfactory neuroepithelium ${ }^{13,22}$. Olfactory and taste dysfunction are common and persistent symptoms of COVID-19 ${ }^{37}$, and a small fraction of hospitalized patients suffer from serious neurological conditions, such as delirium, encephalopathy and stroke ${ }^{37,43}$. The degree to which these neurological effects are due to infection of neuronal cells or the side effects of an inflammatory state are still poorly understood 43,44 . Experimental studies have demonstrated that brain organoids are susceptible to SARS-CoV-2 infection despite the low levels of ACE2 receptor expression ${ }^{44,45}$. Such infection appears to be poorly correlated with levels of ACE2, TMPRSS2, or NRP1 expression ${ }^{44}$. This suggests that neuronal-specific host factors, such as KCNA6, may work independently or in synergy with Spike binding to ACE2 to promote viral entry.

Prior studies have implicated that host ion channels could promote the entry and replication of several viruses ${ }^{46}$. In the case of SARS-CoV-2, preliminary work indicated that pharmacological inhibition of pore $\mathrm{Ca}^{2+}$ channel 2 (TPC2) led to the decreased entry of SARS-CoV-2 pseudovirions ${ }^{47}$. A similar finding using MERS pseudovirions found that knockdown of TPC1 or TPC2 lead to decreased viral entry-an effect explainable by lowered Furin cleavage activity and impaired endosomal motility ${ }^{48}$. Here, we show that FDA-approved KCNA channel blocker 4-AP (dalfampridine) decreased Spike-mediated viral entry (Fig. 4I-K). This suggests a potential mechanism that extends beyond KCNA6 to the KCNA potassium channels more generally, which is further supported by our network analysis and real-world evidence (Fig. 5). As our work indicates that KCNA6 entry promotion and 4-AP inhibition are both dependent on SARS-CoV-2 Spike, it is interesting to note that the presence of $\mathrm{K}^{+}$ions is a critical requirement for a conformational change in the Bunyavirus envelope glycoprotein that precedes viral entry ${ }^{49,50}$. Whether a similar mechanism is at play for SARS-CoV-2 Spike is worthy of further investigation.

Our study used SARS-CoV-2 Spike-pseudotyped lentiviruses in our initial CRISPRa screening. As such, our discovery approach does not consider the potential role of other SARS-CoV-2 components, such as the envelope (E) or nucleocapsid (N) proteins. Our focused membrane sgRNA library likely reduced screen noise, as host factors involved in viral entry are rarely non-membrane-associated, but may not detect some potentially interesting proteins that act indirectly to boost infection.

Taken together, the studies presented here offer insight into SARS-CoV-2 viral tropism, yield potential new targets for drug development or drug repurposing, and present a platform that can be applied to future emerging pathogens to understand viral susceptibility. 


\section{References}

1. Cui, J., Li, F. \& Shi, Z.-L. Origin and evolution of pathogenic coronaviruses. Nat. Rev. Microbiol. 17, 181-192 (2019).

2. Harrison, A. G., Lin, T. \& Wang, P. Mechanisms of SARS-CoV-2 Transmission and Pathogenesis. Trends Immunol. 41, 1100-1115 (2020).

3. Clausen, T. M. et al. SARS-CoV-2 Infection Depends on Cellular Heparan Sulfate and ACE2. Cell 183, 1043-1057.e15 (2020).

4. Wang, R. et al. Genetic Screens Identify Host Factors for SARS-CoV-2 and Common Cold Coronaviruses. Cell 184, 106-119.e14 (2021).

5. Wei, J. et al. Genome-wide CRISPR Screens Reveal Host Factors Critical for SARS-CoV-2 Infection. Cell 184, 76-91.e13 (2021).

6. Zang, R. et al. Cholesterol 25-hydroxylase suppresses SARS-CoV-2 replication by blocking membrane fusion. Proc. Natl. Acad. Sci. U. S. A. 117, 32105-32113 (2020).

7. Yeung, M. L. et al. Soluble ACE2-mediated cell entry of SARS-CoV-2 via interaction with proteins related to the renin-angiotensin system. Cell 184, 2212-2228.e12 (2021).

8. Baggen, J. et al. Genome-wide CRISPR screening identifies TMEM106B as a proviral host factor for SARS-CoV-2. Nat. Genet. 53, 435-444 (2021).

9. Daniloski, Z. et al. Identification of Required Host Factors for SARS-CoV-2 Infection in Human Cells. Cell 184, 92-105.e16 (2021).

10. Hoffmann, H.-H. et al. TMEM41B Is a Pan-flavivirus Host Factor. Cell 184, 133148.e20 (2021). 
11. Schneider, W. M. et al. Genome-Scale Identification of SARS-CoV-2 and Pancoronavirus Host Factor Networks. Cell 184, 120-132.e14 (2021).

12. Bailey, A. L. \& Diamond, M. S. A Crisp(r) New Perspective on SARS-CoV-2 Biology. Cell 184, 15-17 (2021).

13. Cantuti-Castelvetri, L. et al. Neuropilin-1 facilitates SARS-CoV-2 cell entry and infectivity. Science 370, 856-860 (2020).

14. Daly, J. L. et al. Neuropilin-1 is a host factor for SARS-CoV-2 infection. Science $\mathbf{3 7 0 ,}$ 861-865 (2020).

15. Weinreich, D. M. et al. REGN-COV2, a Neutralizing Antibody Cocktail, in Outpatients with Covid-19. N. Engl. J. Med. 384, 238-251 (2021).

16. Polack, F. P. et al. Safety and Efficacy of the BNT162b2 mRNA Covid-19 Vaccine. N. Engl. J. Med. 383, 2603-2615 (2020).

17. Hoffmann, M. et al. SARS-CoV-2 variants B.1.351 and P.1 escape from neutralizing antibodies. Cell 184, 2384-2393.e12 (2021).

18. Dejnirattisai, W. et al. Antibody evasion by the P.1 strain of SARS-CoV-2. Cell 184, 2939-2954.e9 (2021).

19. Wang, P. et al. Antibody resistance of SARS-CoV-2 variants B.1.351 and B.1.1.7. Nature 593, 130-135 (2021).

20. Abu-Raddad, L. J., Chemaitelly, H., Butt, A. A., \& National Study Group for COVID19 Vaccination. Effectiveness of the BNT162b2 Covid-19 Vaccine against the B.1.1.7 and B.1.351 Variants. N. Engl. J. Med. (2021) doi:10.1056/NEJMc2104974.

21. Wall, E. C. et al. Neutralising antibody activity against SARS-CoV-2 VOCs B.1.617.2 and B.1.351 by BNT162b2 vaccination. Lancet Lond. Engl. (2021) doi:10.1016/S0140-6736(21)01290-3. 
22. Meinhardt, J. et al. Olfactory transmucosal SARS-CoV-2 invasion as a port of central nervous system entry in individuals with COVID-19. Nat. Neurosci. 24, 168-175 (2021).

23. Finkelshtein, D., Werman, A., Novick, D., Barak, S. \& Rubinstein, M. LDL receptor and its family members serve as the cellular receptors for vesicular stomatitis virus. Proc. Natl. Acad. Sci. 110, 7306-7311 (2013).

24. The GTEx Consortium. The GTEx Consortium atlas of genetic regulatory effects across human tissues. Science 369, 1318-1330 (2020).

25. Puelles, V. G. et al. Multiorgan and Renal Tropism of SARS-CoV-2. N. Engl. J. Med. 383, 590-592 (2020).

26. Lindner, D. et al. Association of Cardiac Infection With SARS-CoV-2 in Confirmed COVID-19 Autopsy Cases. JAMA Cardiol. 5, 1281 (2020).

27. de Melo, G. D. et al. COVID-19-related anosmia is associated with viral persistence and inflammation in human olfactory epithelium and brain infection in hamsters. Sci. Transl. Med. 13, eabf8396 (2021).

28. Uhlén, M. et al. Proteomics. Tissue-based map of the human proteome. Science 347, 1260419 (2015).

29. Liao, M. et al. Single-cell landscape of bronchoalveolar immune cells in patients with COVID-19. Nat. Med. 26, 842-844 (2020).

30. Mehdipour, A. R. \& Hummer, G. Dual nature of human ACE2 glycosylation in binding to SARS-CoV-2 spike. Proc. Natl. Acad. Sci. U. S. A. 118, (2021).

31. Dinnon, K. H. et al. A mouse-adapted model of SARS-CoV-2 to test COVID-19 countermeasures. Nature 586, 560-566 (2020). 
32. Olender, T. et al. The human olfactory transcriptome. BMC Genomics 17, 619 (2016).

33. Durante, M. A. et al. Single-cell analysis of olfactory neurogenesis and differentiation in adult humans. Nat. Neurosci. 23, 323-326 (2020).

34. Hikmet, F. et al. The protein expression profile of ACE2 in human tissues. Mol. Syst. Biol. 16, e9610 (2020).

35. Lukassen, S. et al. SARS-CoV-2 receptor ACE2 and TMPRSS2 are primarily expressed in bronchial transient secretory cells. EMBO J. 39, e105114 (2020).

36. Aguiar, J. A. et al. Gene expression and in situ protein profiling of candidate SARSCoV-2 receptors in human airway epithelial cells and lung tissue. Eur. Respir. J. 56, (2020).

37. Ellul, M. A. et al. Neurological associations of COVID-19. Lancet Neurol. 19, 767783 (2020).

38. Hayes, K. C. The use of 4-aminopyridine (fampridine) in demyelinating disorders. CNS Drug Rev. 10, 295-316 (2004).

39. Zhang, Z. et al. Cleavage of tau by asparagine endopeptidase mediates the neurofibrillary pathology in Alzheimer's disease. Nat. Med. 20, 1254-1262 (2014).

40. Gordon, D. E. et al. Comparative host-coronavirus protein interaction networks reveal pan-viral disease mechanisms. Science 370, (2020).

41. Zimniak, M. et al. The serotonin reuptake inhibitor Fluoxetine inhibits SARS-CoV-2 in human lung tissue. Sci. Rep. 11, 5890 (2021).

42. Chen, R. E. et al. Resistance of SARS-CoV-2 variants to neutralization by monoclonal and serum-derived polyclonal antibodies. Nat. Med. 27, 717-726 (2021). 
43. Helms, J. et al. Neurologic Features in Severe SARS-CoV-2 Infection. N. Engl. J. Med. 382, 2268-2270 (2020).

44. Song, E. et al. Neuroinvasion of SARS-CoV-2 in human and mouse brain. J. Exp. Med. 218, e20202135 (2021).

45. Ramani, A. et al. SARS-CoV-2 targets neurons of 3D human brain organoids. EMBO J. 39, e106230 (2020).

46. Charlton, F. W. et al. Ion Channels as Therapeutic Targets for Viral Infections: Further Discoveries and Future Perspectives. Viruses 12, E844 (2020).

47. Ou, X. et al. Characterization of spike glycoprotein of SARS-CoV-2 on virus entry and its immune cross-reactivity with SARS-CoV. Nat. Commun. 11, 1620 (2020).

48. Gunaratne, G. S., Yang, Y., Li, F., Walseth, T. F. \& Marchant, J. S. NAADPdependent $\mathrm{Ca} 2+$ signaling regulates Middle East respiratory syndrome-coronavirus pseudovirus translocation through the endolysosomal system. Cell Calcium 75, 30$41(2018)$.

49. Punch, E. K. et al. Potassium is a trigger for conformational change in the fusion spike of an enveloped RNA virus. J. Biol. Chem. 293, 9937-9944 (2018).

50. Hover, S. et al. Modulation of Potassium Channels Inhibits Bunyavirus Infection. J. Biol. Chem. 291, 3411-3422 (2016).

\section{Acknowledgments}

We are grateful to members of the laboratories of Dr. Le Cong and Dr. Michael Cleary. We are grateful to Dr. Julien Sage for helpful discussion and support on understanding screen results and validation of host factors; to Dr. Joseph Wu, Dr. Mingqiang Wang, Dr. Masataka Nishiga for discussion on single-cell gene expression analysis; to Dr. Weishan Huang and Dr. Tianyi Zhang for helpful discussion on SARSCoV-2 virus validation. 
A

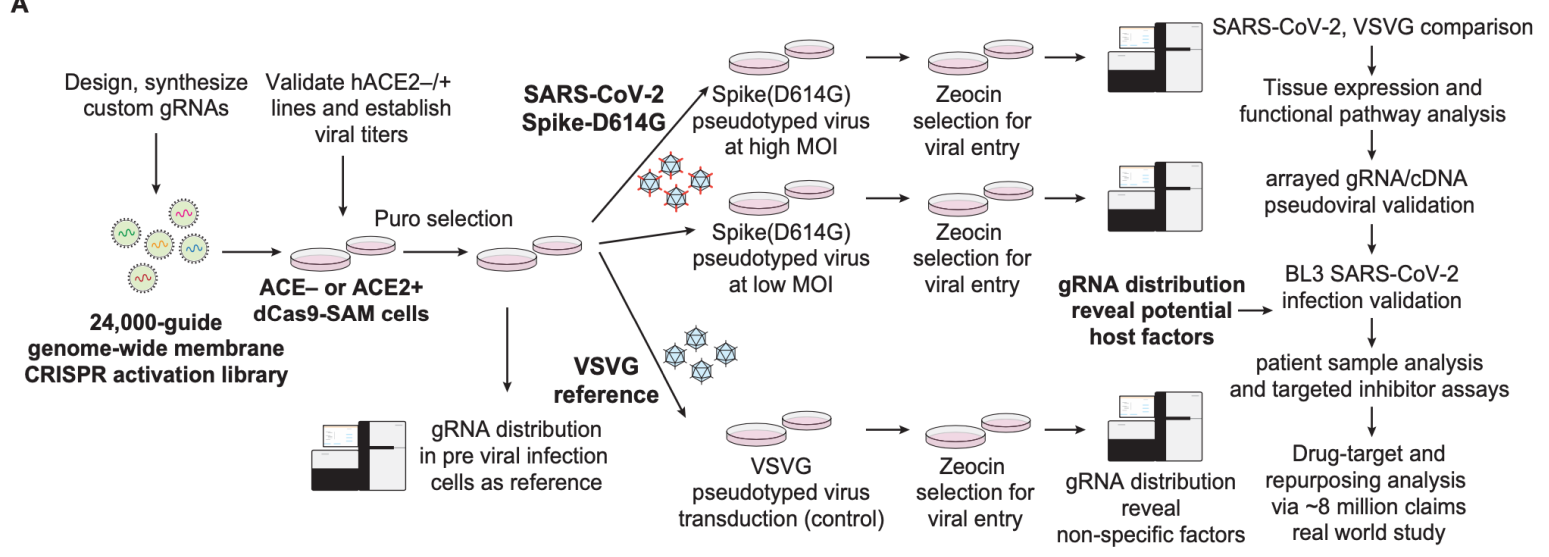

B

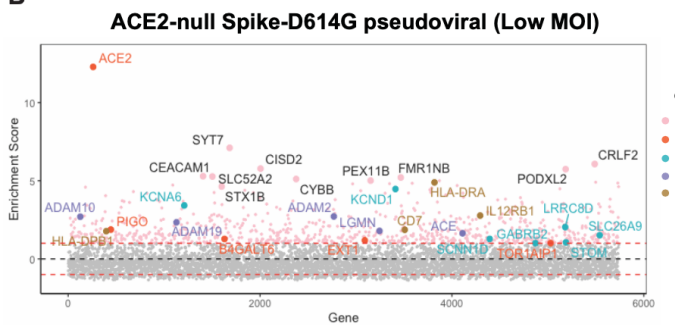

D

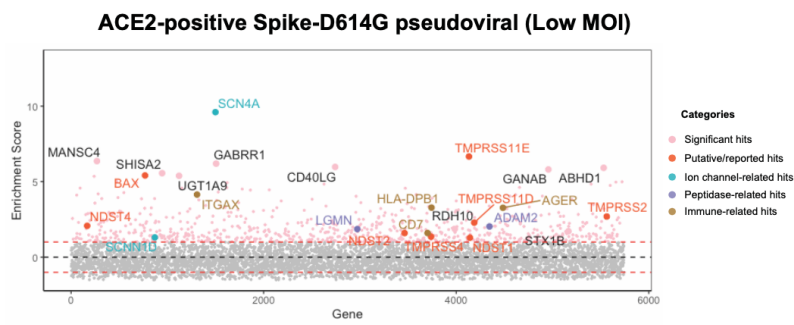

$\mathbf{F}$

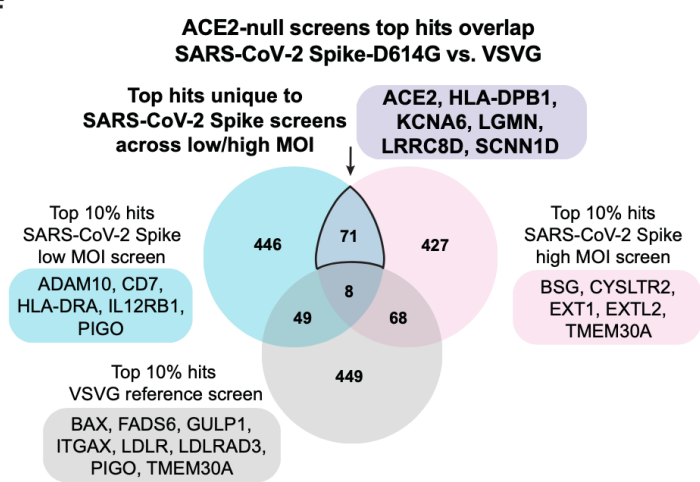

C

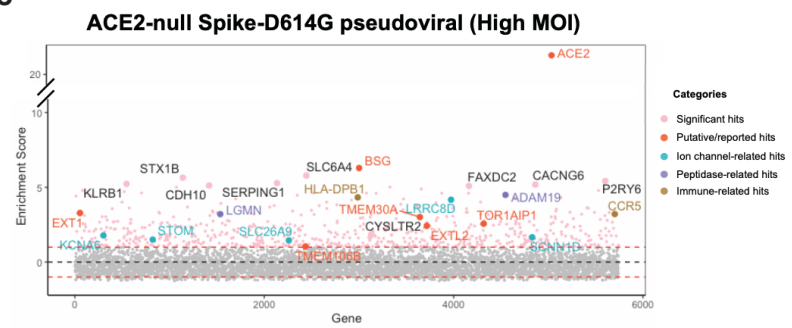

E

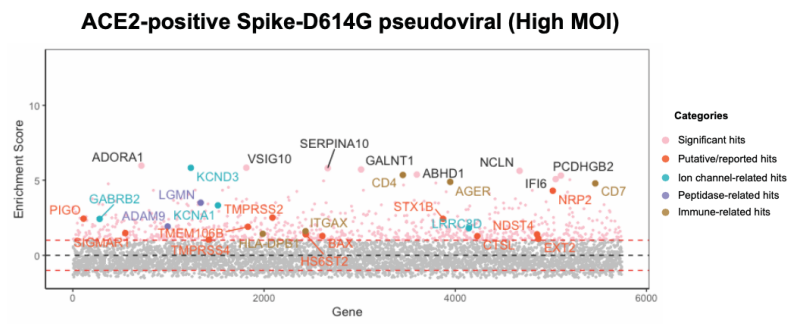

G

ACE2-positive screens top hits overlap SARS-CoV-2 Spike-D614G vs. VSVG

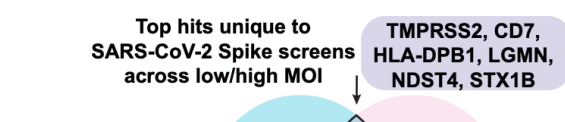

Fig. 1. Membrane-focused CRISPRa screening identifies potential host factors involved in Spike-dependent SARS-CoV-2 virus entry. (A) Screen pipeline showing different conditions used (ACE2-null, ACE2-positive, at low or high MOI, with VSVG references), downstream analyses and validation workflow. (B-E) Enrichment scores of CRISPRa screen across different conditions with top hits highlighted and colored by their functional categories. (F-G) Differential analysis of top 10\% hits from SARS-CoV-2 Spike and reference VSVG screens. The unique hits in SARS-CoV-2 screens identify putative virus-specific host factors. 
A TPM $^{0.0} \quad$\begin{tabular}{lllll}
3.4 & 19 & 87 & $3.90+2$ & $1.70+3$ \\
\hline
\end{tabular}

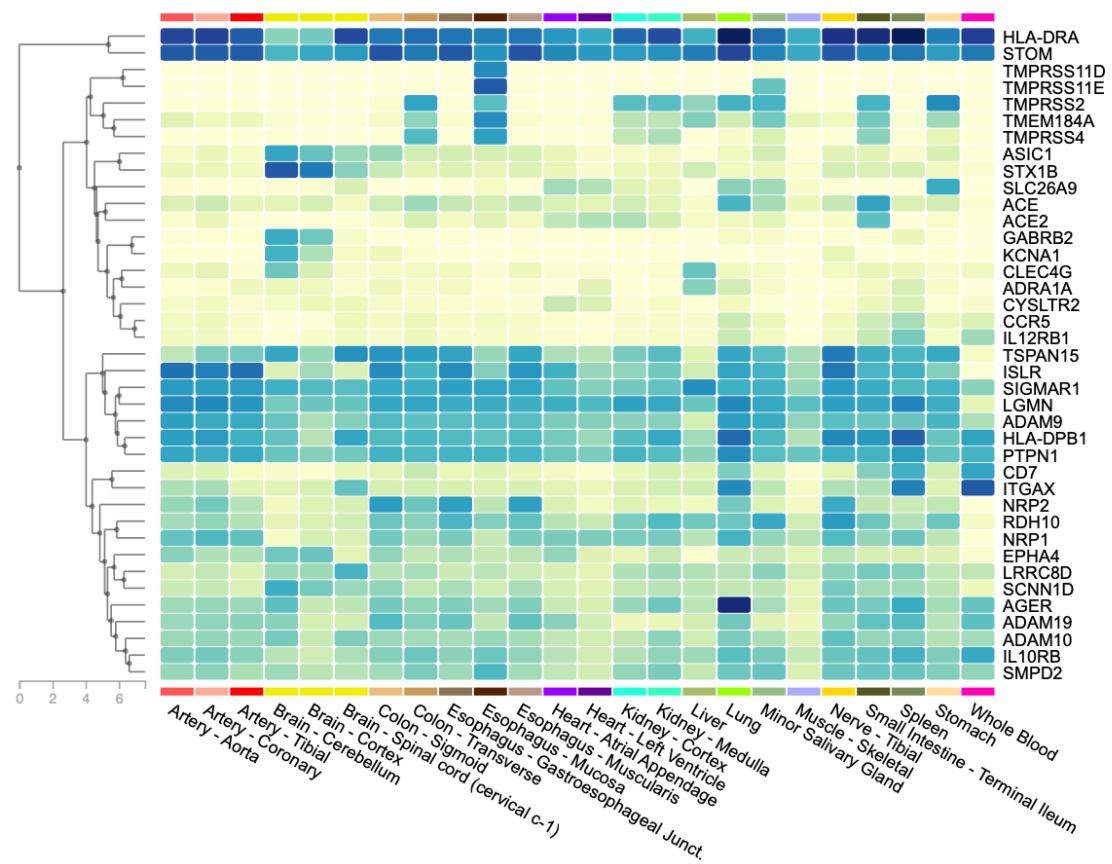

B Human body expression profiles of top screen hits

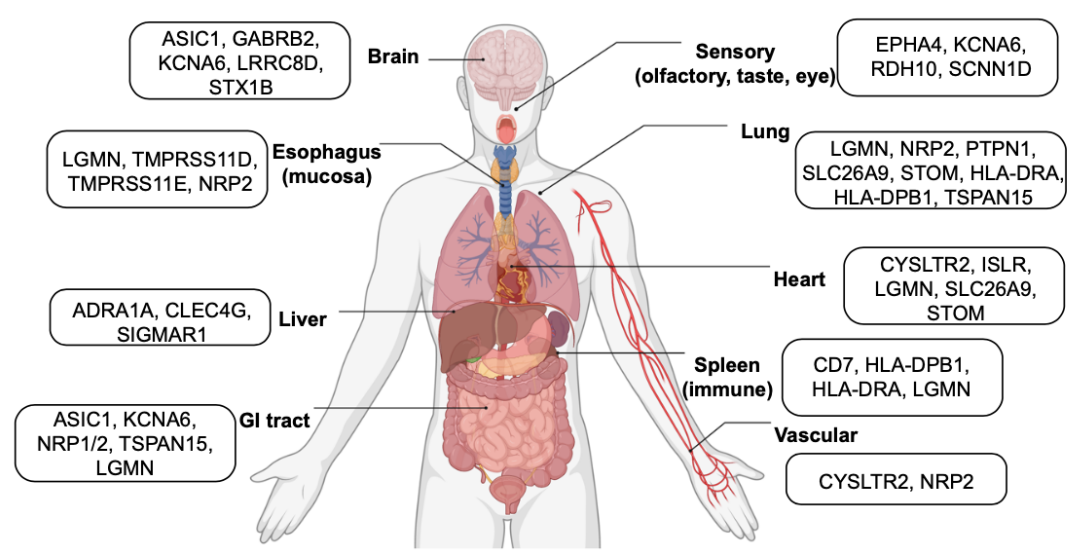

C

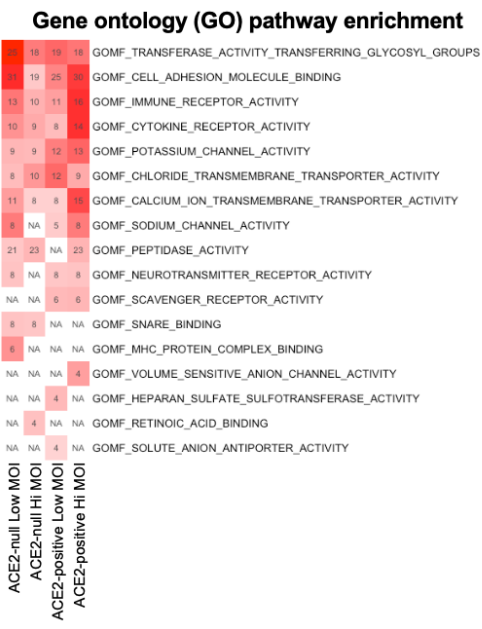

D
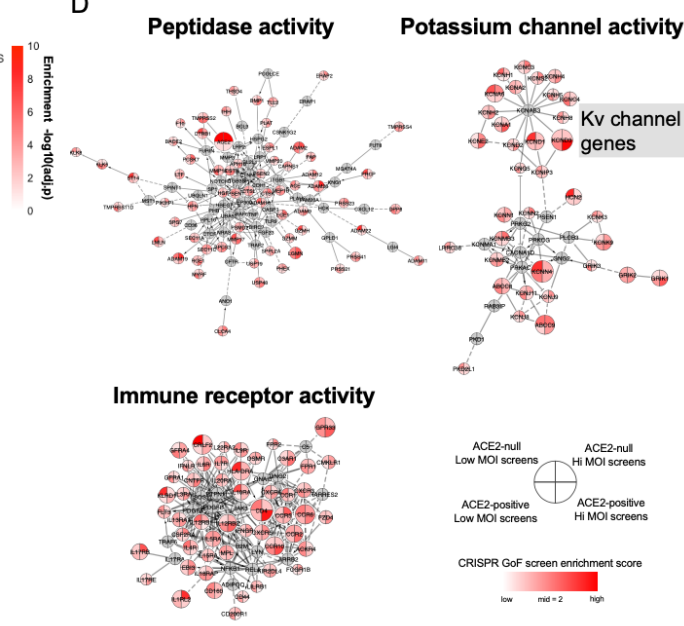

Fig. 2. Tissue expression and functional enrichment analysis of top-ranking genes promoting viral entry. (A) Heatmap showing the overall human tissue 
expression patterns of top-ranking genes using GTEX v8 dataset. (B) Tissue expression body map of top-ranking genes, showing putative host genes expressed in the respiratory, neuronal, cardiovascular, liver, gastrointestinal and immune systems. (C) Gene set overlap analysis using gene ontology (GO) on top $10 \%$ of hits from each screen condition. The top GO terms of each screen condition were selected for visualization. (D) Selected functional network clusters involved with the top GO terms. Colored nodes are significant hits identified from the screen and grey nodes are the connecting nodes. The enrichment score of a gene in each category of screens is indicated by color scale within the node. Notable genes within the same family are highlighted. 
A

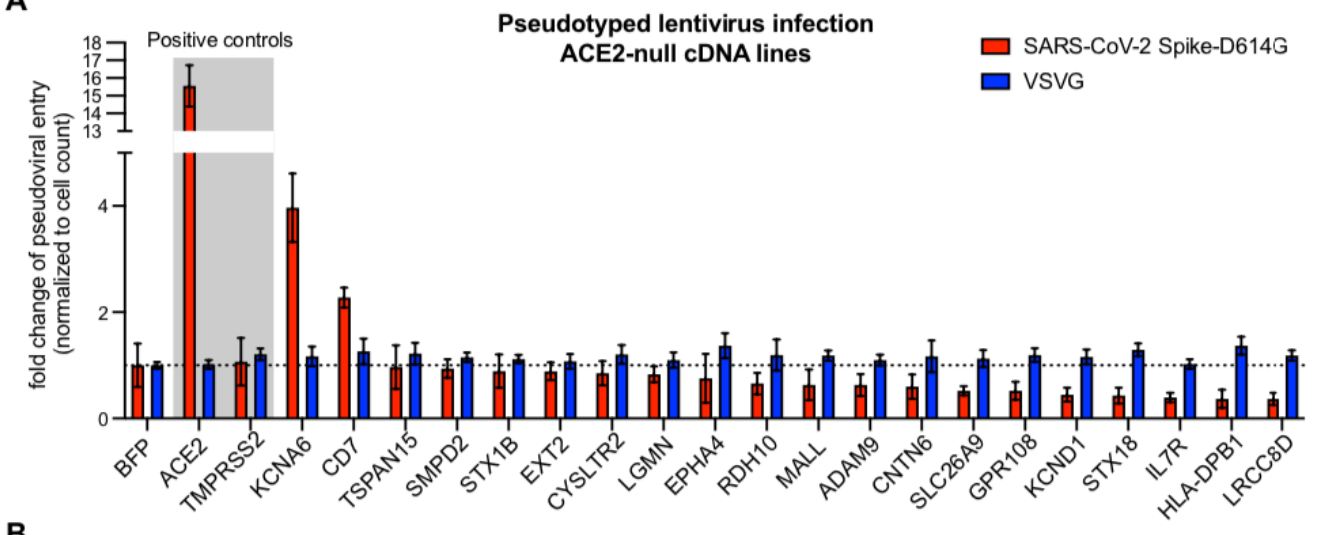

в

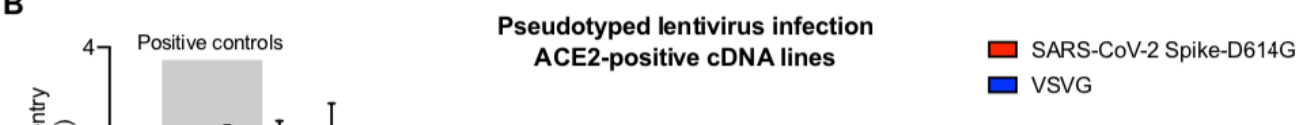

Replicating SCoV-2 Spike-pseudotyped VSV infection

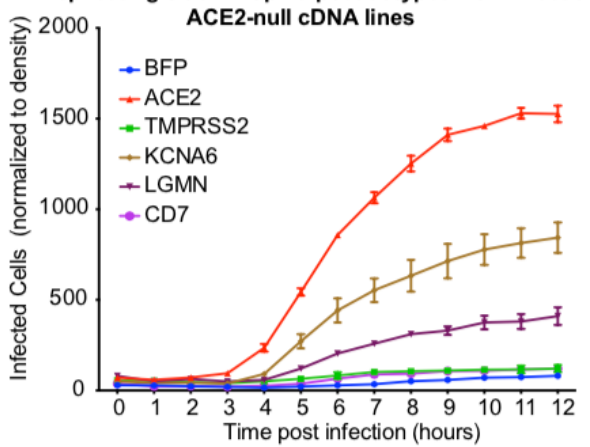

E

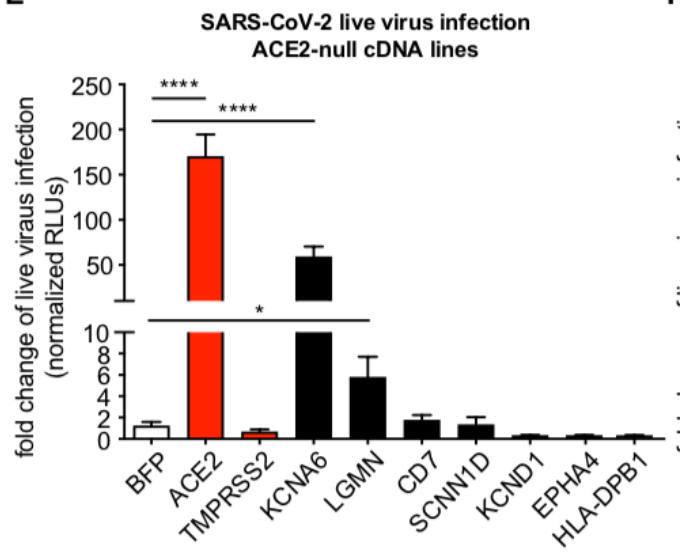

Replicating SCoV-2 Spike-pseudotyped VSV infection

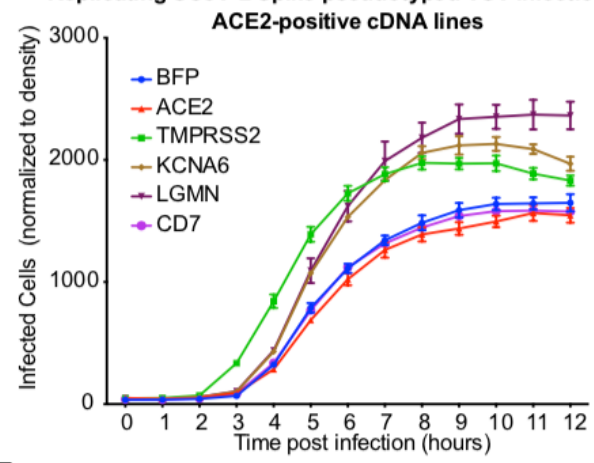

$\mathbf{F}$

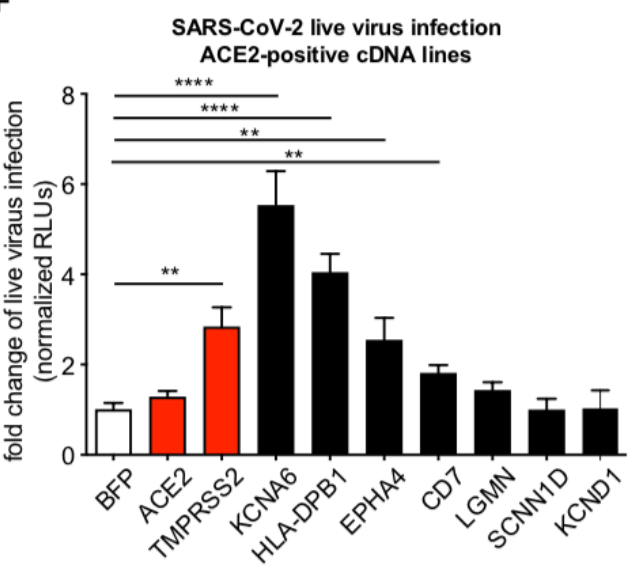

Fig. 3. Validation of top-ranked genes using pseudoviral and replicationcompetent SARS-CoV-2 assays. (A-B) Arrayed validation of top hits in cDNA 
overexpressing cell lines of individual genes using SARS-CoV-2 Spike-D614G pseudotyped lentiviral assay. The control VSVG-pseudotyped lentivirus results are shown side-by-side. Results are normed to the BFP control for each respective pseudotype. Data from two independent experiments. (C-D) Arrayed validation using time-lapse imaging of replicating SARS-CoV-2 Spike-pseudotyped VSV infection in cDNA overexpression cell lines. Data from two independent experiments. (E-F) Validation of top-ranked genes using SARS-CoV-2-nLuc virus infection. Data from three independent experiments. Data plotted represents the mean with SEM. Statistical analyses were performed via two-tailed t-test, ${ }^{*}, p<0.05 ;{ }^{* *}, p<0.01 ;{ }^{* * *}, p<0.001 ;{ }^{* * * *}$, $p<0.0001$. 


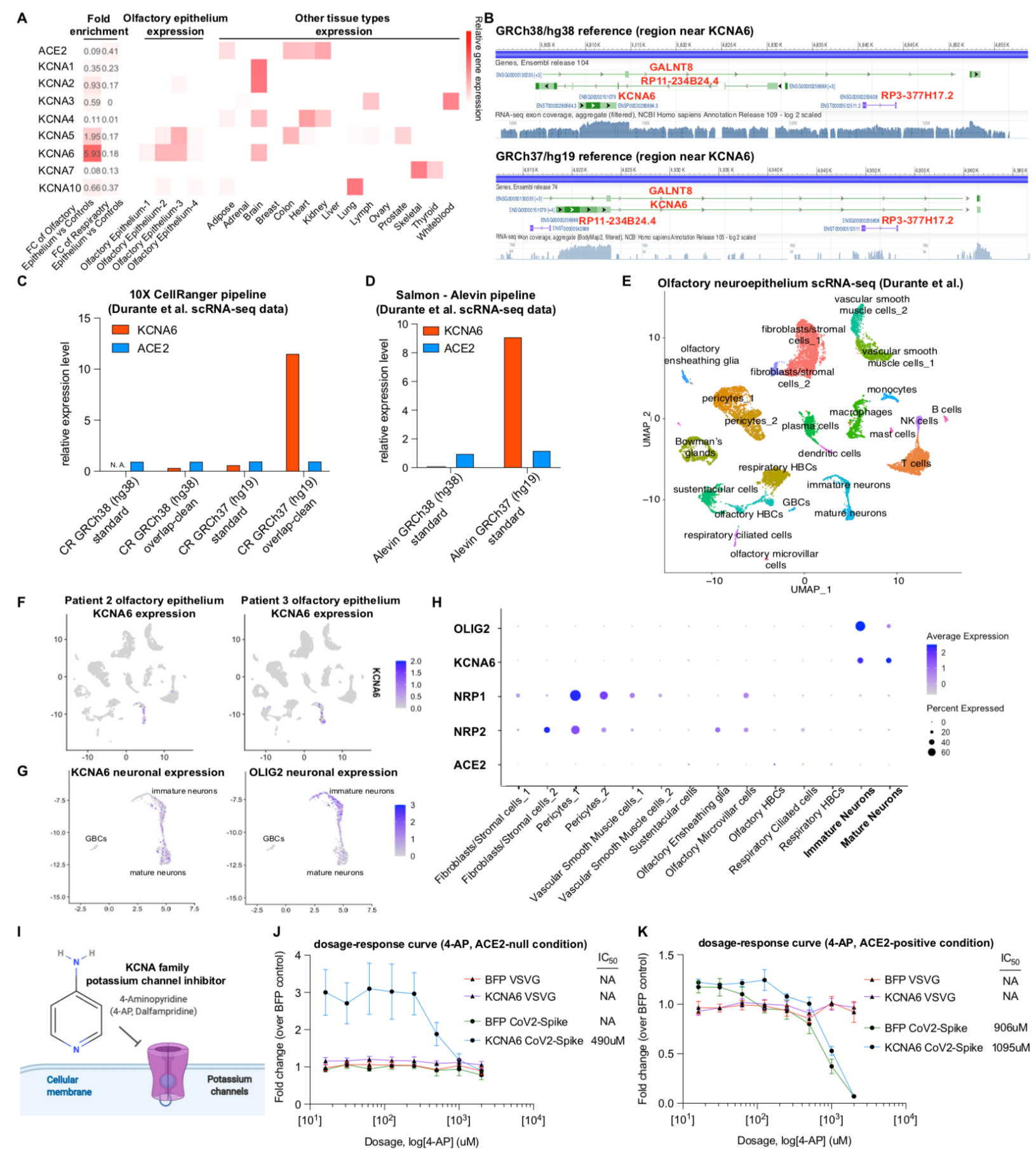

Fig. 4. KCNA6 is highly expressed in nasal/olfactory neurons and is a druggable target for inhibiting SARS-CoV-2 viral entry. (A) Expression of ACE2 and KCNA genes across human tissues. Left two columns show the fold change of olfactory/respiratory expression vs control tissue expression. Columns in the middle and right show the expression levels in 4 olfactory epithelial samples and 15 control tissues (from Olender et al.). (B) KCNA6 genome annotations in the GRCh38 (hg38) and GRCh37 (hg19) references using NCBI Genome Data Viewer. (C) Expression of ACE2 and KCNA6 in the single-cell RNA-seq data of olfactory neuroepithelium using different versions of genome references (from Durante et al.). Cell Ranger 6.0 was used for all 
alignments and the expression was calculated by averaging the ACE2/KCNA6 expression in all cells and normalized to the ACE2 expression from the standard GRCh38 reference genome. (D) Expression of ACE2/KCNA6 using the Salmon - Alevin pipeline, calculated similarly as in panel C. The standard GRCh37/GRCh38 genome references were used. (E) UMAP depicting the olfactory epithelial cell types from two patients. The cell cluster identities were based on Durante et al. (F) UMAPs depicting the expression levels of KCNA6 in individual patients. (G) Focused UMAPs of the neuronal populations showing co-expression of KCNA6 and OLIG2, a reported marker for virus-infected neuronal cells in COVID19 patient olfactory neuroepithelium. (H) Dot plot expression of selected genes in olfactory epithelial cell types. Sizes of the dots indicate the proportion of cells in the respective cell type having greater-than-zero expression while the color indicates the mean expression. (I-K) FDA-approved compound 4-Aminopyridine (4-AP, dalfampridine) is a broad-spectrum potassium channel inhibitor (I). Inhibitor assays performed in ACE2-null (J) or ACE2-positive (K) conditions, measuring SARS-CoV-2 spike-D614G or VSV-G pseudotyped lentiviruses infection efficiencies in KCNA6 overexpression or control BFP lines treated with different doses of 4-AP, with measured $\mathrm{IC}_{50}$ to the right. Results are normed to the BFP control/vehicle for each respective pseudotype. Data from two independent experiments. Data plotted represents the mean with SEM. 
A

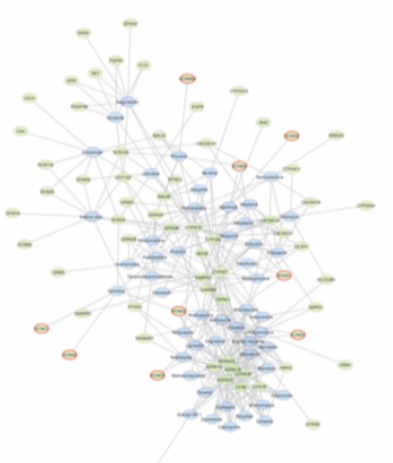

C

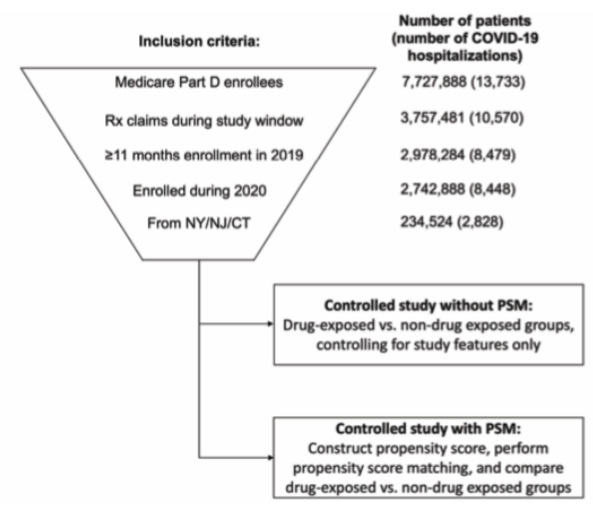

$E$

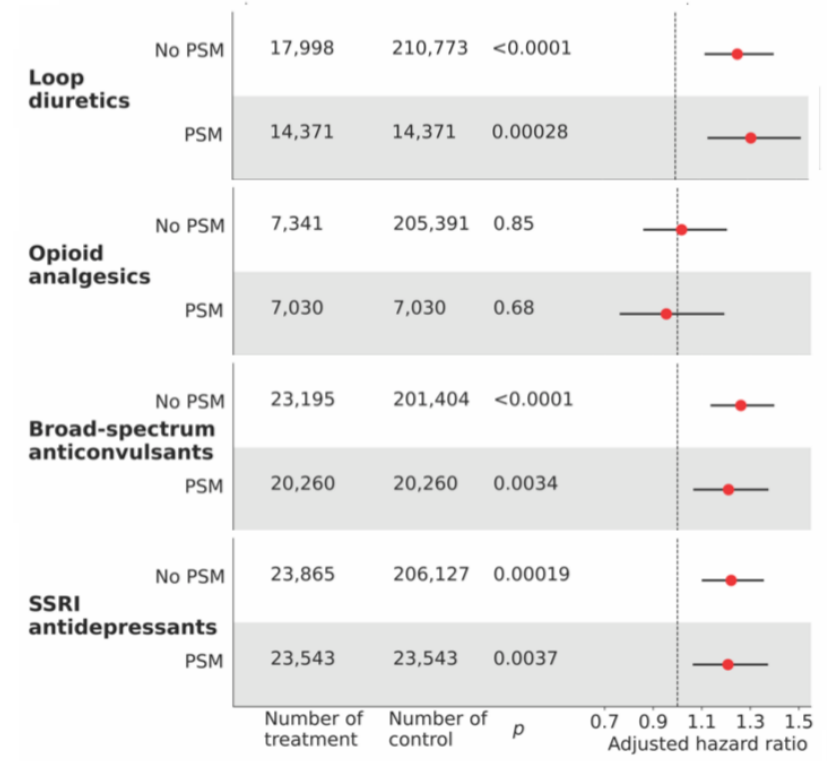

B

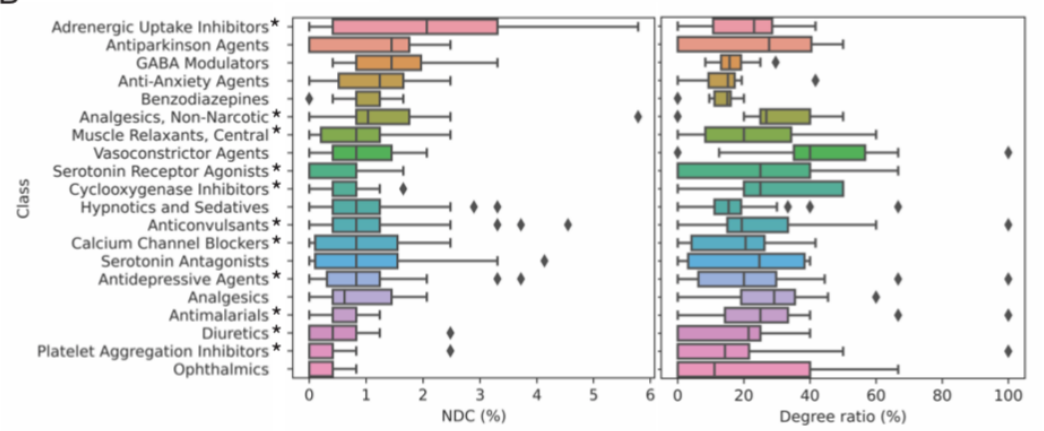

D

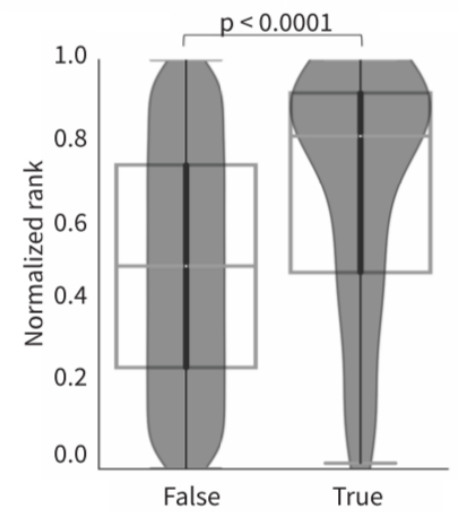

F

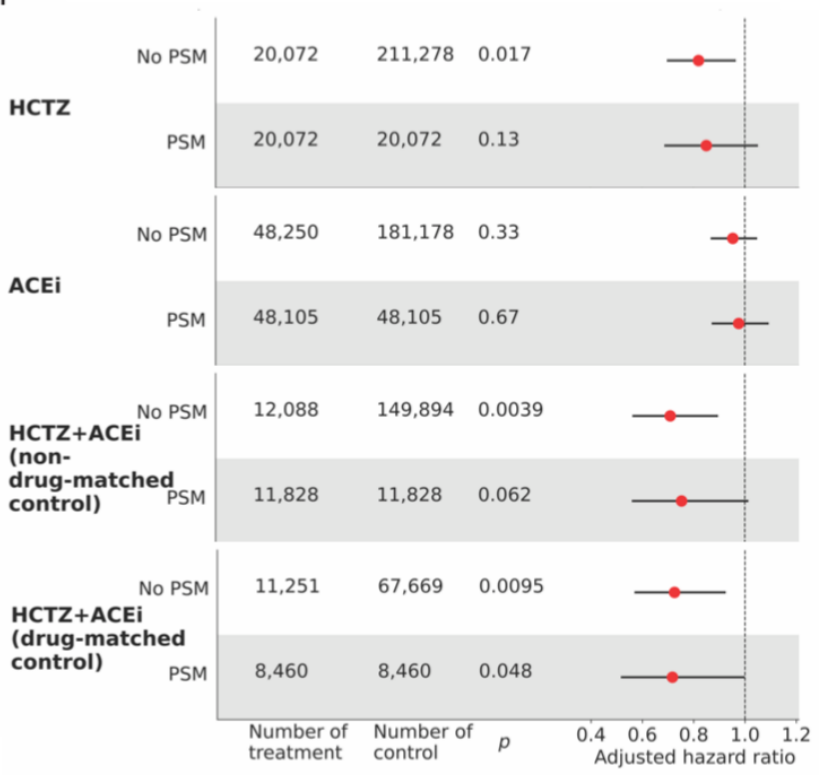

Fig. 5. Network analysis identifies drugs that target top screen hits, and retrospective analysis of patient claims provides real world evidence for enriched drug categories. (A) Overview of the drug-target interaction network, showing an induced subgraph of the 50 highest ranked compounds (drugs in blue; screen hits in green; potassium channel genes outlined in red). (B) Top drug classes enriched in hits from the interaction network model by NDC and degree ratio with respect to screen hits. Asterisks indicate drug classes with at least one member targeting a potassium channel. (C) Controlled study design for COVID-19 hospitalization from pharmaceutical claims data. (D) Drugs associated with COVID-19 hospitalization in the unmatched study rank highly in the drug-target interaction network. (E-F) Real world evidence for 
bioRxiv preprint doi: https://doi.org/10.1101/2021.07.01.450475; this version posted July 1, 2021. The copyright holder for this preprint (which was not certified by peer review) is the author/funder. All rights reserved. No reuse allowed without permission.

associations between ion-channel-targeting drug classes identified in the screen and increased $(E)$ and decreased $(F)$ risk of COVID-19 hospitalization in propensity-scorematched subjects. 